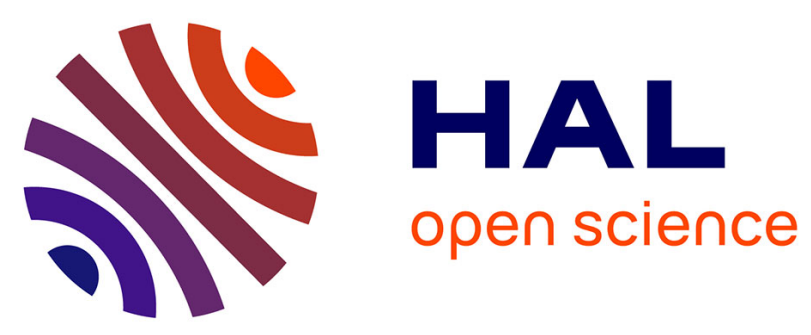

\title{
Anthropologie et industrie
}

Denis Guigo

\section{- To cite this version:}

Denis Guigo. Anthropologie et industrie. Journal des anthropologues, 1991, 43-44 (Ethnologie de l'entreprise, Monique Selim et Kurumi Sugita, dir.), pp.39-43. hal-00460399

\section{HAL Id: hal-00460399 \\ https://hal.science/hal-00460399}

Submitted on 28 Feb 2010

HAL is a multi-disciplinary open access archive for the deposit and dissemination of scientific research documents, whether they are published or not. The documents may come from teaching and research institutions in France or abroad, or from public or private research centers.
L'archive ouverte pluridisciplinaire HAL, est destinée au dépôt et à la diffusion de documents scientifiques de niveau recherche, publiés ou non, émanant des établissements d'enseignement et de recherche français ou étrangers, des laboratoires publics ou privés. 


\title{
ANTHROPOLOGIE ET INDUSTRIE
}

\author{
Denis GUIGO \\ (Ecole Polytechnique-CRG, CNRS-LAU)
}

Anthropologie et industrie : deux termes que l'on ne rapproche pas spontanément. Les premières enquêtes ethnographiques en milieu industriel, réalisées il y a plus de soixante ans, donnèrent pourtant naissance au courant de pensée américain dit "human relation school", qui marqua profondément la réflexion postérieure sur le travail en usine. Il est vrai qu'il s'agissait là d'une anthropologie appliquée, d'inspiration fonctionnaliste, qui dialoguait sans doute plus aisément avec la psychosociologie qu'avec l'ethnologie "traditionnelle". Ultérieurement, bon nombre de travaux d'anthropologie industrielle s'inscrivent dans deux pradigmes principaux: une vision critique du système de production capitaliste (une orientation "anti-appliquée", en quelque sorte...), et une perspective plutôt comparative (notamment des "cross-cultural studies"), mais qui s'attachait à des niveaux très variables de l'organisation industrielle (le pays, la branche, la région, la technologie...) (1).

En somme, l'anthropologie industrielle existe certes, mais son orientation et ses objets sont multiformes; de plus, l'existence d'un courant grandissant de recherche appliquée ajoute à la complexité du

1. Pour une histoire de l'anthropologie industrielle, voir Michael BURAWOY, "The Anthropology of Industrial Work", Annual Review of Anthropology, Palo Alto (California), $8: 231-266$, 1979 ; Jean COPANS et Bernard BERNIER, introduction au numéro "Travail, industries et classes ouvrières", Anthropologie et sociétés, Montréal (Québec), Université Laval, vol. 10, $n^{\circ} 1: 1-9,1986$; et surtout Carol HOLZBERG et Maureen GIOVANNINI, "Anthropology and Industry: Reappraisal and New Directions", Annual Review of Anthropology, $10: 317-360,1981$. 
tableau. Si la méthodologie utilisée se réclame souvent de l'ethnographie de terrain, les résultats d'enquête peuvent intéresser, selon le cas, le chef d'entreprise, les syndicats, le commanditaire d'une étude, la communauté scientifique d'une discipline (pas forcément l'ethnologie), ou d'autres acteurs concernés à un titre ou à un autre par l'évolution d'une branche industrielle. Cette diversité ne facilite pas la "visibilité" de l'anthropologie industrielle dans le champ scientifique, d'autant que certains chercheurs jugent que l'heure n'est pas aux ambitions théoriques, et préfèrent pour l'instant accumuler des matériaux. Il ne m'appartient bien évidemment pas de juger les différentes manières de conjuguer anthropologie et industrie ; je me limiterai ici à proposer quelques réflexions à partir des recherches que j'ai pu mener récemment dans une entreprise automobile française (1985) (2), une compagnie sidérurgique argentine (1985-1986) (3), l'entreprise d'électricité du Grand Buenos Aires (1986-1987) (4) et une mairie argentine (1987-1988) (5).

C'est surtout la question de l'ambiguïté éventuelle du rapport entre ethnologie "appliquée" et ethnologie "fondamentale" que je voudrais évoquer ici. Les problèmes de la "recherche-action" ont été traités dans nombre de colloques et de publications - souvent dans le champ d'autres disciplines, mais pas uniquement, comme l'a montré le numéro de la revue Anthropologie et Sociétés consacré au thème "Comprendre et Modifier" (6); les questions soulevées par la relation entre commanditaires d'études et ethnologues ne semblent pas absolument spécifiques. S'il est clair que les recherches appliquée et fondamentale

2. Cf. D. GUIGO, "L'empire du consensus" (deux épisodes), Annales des Mines, Série Gérer et Comprendre, $\mathrm{n}^{\circ} 6: 19-26$ et $\mathrm{n}^{\circ} 7: 24-33$, Paris, 1987.

3. Cf. D. GUIGO, "Automatisation et enjeux de gestion", Travail et travailleurs du TiersMonde, Les Cahiers de l'Orstom, $n^{\circ} 4: 3-48$, Paris, 1987.

4. Cf. D. GUIGO, "El servicio público frente a la crisis : el caso de SEGBA en el Gran Buenos Aires", communication au colloque La Región Metropolitana Buenos Aires, CNRS et Gouvernement de la Province de Buenos Aires, Mar del Planta, avril 1989.

5. Cf. D. GUIGO, "Gobernar un Municipio", communication au colloque Governo Municipal na América Latina, Universidad Federal do Rio Grande Do Sul, Porto Alegre, Brésil, décembre 1989.

6. "Comprendre et modifier", Anthropologie et Sociétés, vol. 8, $\mathrm{n}^{\circ} 3$, Université Laval (Québec), 1984. 
forment deux démarches différentes, elles peuvent cependant aller de pair, mieux encore, se fertiliser l'une l'autre au cours d'un même travail de terrain, dans la mesure où les termes de l'échange entre le chercheur et le milieu étudié sont clairement définis (7). Par exemple, à la fin de l'étude de l'entreprise automobile mentionnée ci-dessus (étude demandée et financée par cette société), un rapport fut remis à mes interlocuteurs ; il leur donna satisfaction, non en tant que vérité définitive sur le sujet (les innovations introduites par le "management participatif"), mais en tant que point de vue complémentaire, susceptible d'être pris en compte par les décideurs. Un an et demi plus tard, un autre texte (Cf. note 2), plus éloigné des préoccupations de gestion et axé sur une analyse "néofoucaldienne" de l'exercice du pouvoir dans les ateliers, fut publié par une revue scientifique (en respectant l'anonymat de l'entreprise) puis repris par une revue professionnelle. Les deux textes étaient certes différents; chaque partenaire trouva son compte dans l'affaire.

Point n'est besoin de souligner que le travail de terrain permet la moisson d'une masse considérable d'informations qui pourront plus tard être valorisées; c'est ainsi qu'un relevé du vocabulaire d'adresse au bureau a été réalisé à l'occasion de la même enquête, et exploité ultérieurement (8). D'autre part, outre les publications mentionnées en notes 3, 4 et 5 , qui intéressaient au premier chef les personnes concernées par l'automatisation sidérurgique, la distribution d'électricité ou l'administration municipale, les enquêtes correspondantes fournirent des résultats susceptibles d'intéresser un public plus large, sur des thèmes où l'approche ethnographique apparaît pertinente par sa capacité à articuler plusieurs dimensions d'objets sociaux complexes (9).

7. Plus généralement; GUILLE-ESCURET a récemment noté : "Il a maintes fois été répété qu'une recherche appliquée ne saurait se développer sans la progression conjointe de la recherche fondamentale. C'est indubitable, mais n'oublie-t-on pas que l'inverse est aussi vrai ?" ("Une recherche perdue en son temps : l'ethnologie inappliquée", L'Homme, $\mathrm{n}^{\circ} 115$ : 110 , Paris, 1990.

8. "L'Adresse au bureau", communication présentée au colloque Travail et pratiques langagietes, PIRTTEM-CNRS, Paris, avril 1989. Une version remaniée paraîtra dans L'Homme en 1991.

9. Cf. différentes publications concernant respectivement l'hyperinflation ("Cohabiter avec un monstre. L'hyperinflation en Argentine", Annales des Mines, série Gérer et Comprendre, $n^{\circ} 8$ : 77-82, Paris, 1987; "Gérer en hyperinflation", idem, $n^{\circ} 13: 59-73,1988$; 
Les recherches sur les terrains argentins mentionnés ici (l'entreprise sidérurgique, la compagnie d'électricité et la mairie) ne furent pas rémunérés par les organisations en question, mais financées par une bourse de doctorat du CNRS ; dans chaque cas, il s'agissait d'abord de faire accepter - ou tolérer - ma présence par les principaux acteurs (dirigeants et syndicats notamment), en étant attentif aux demandes explicites ou implicites qui émanaient de mes interlocuteurs de différents niveaux. En effet, comme dans une société "exotique" sans doute, les gens ne parlaient pas - ou pas seulement - au chercheur "pour le plaisir", mais en fonction de leurs enjeux. Plus généralement, considérer la relation au terrain comme un matériau de recherche est une démarche intéressante du point de vue heuristique (10).

Le clivage ethnologie appliquée/ethnologie fondamentale semble donc pouvoir être reformulé en termes de demande explicite ou implicite, en distinguant, au sein des demandes explicites, selon que le demandeur coïncide ou non avec le financeur. Pas plus qu'ailleurs, on ne dispose de recettes pour gérer la relation au terrain (11). Tout au moins peut-on souligner l'importance de la liberté de mouvement dont doit disposer l'ethnologue pour récolter ses matériaux dans le milieu complexe et stratifié des organisations modernes. Par exemple, s'il peut être intéressant d'occuper parfois un poste de travail, il faut aussi pouvoir en sortir, sauf à être cantonné dans les postes subalternes et à perdre ainsi l'intelligence d'un aspect essentiel du fonctionnement d'une entreprise :

\footnotetext{
"Hyperinflation en Argentine", Le Monde, 17 mai 1989), les usagers clandestins du réseau d'electricité "Leeches" in the Electrical Network : The Crisis in the Buenos Aires Suburbs", Flux, CNRS-GRD Réseaux, $\mathrm{n}^{\circ} 1: 57-76$ ), printemps 1990 , et la vie dans une municipalité argentine (Poderes en la Administración Municipal, à paraître aux Editions Centre Editor de América Latina, Buenos Aires).

10. "...l'interaction observateur-observé est un problème incontournable mais plutôt que de le considérer comme un obstacle à la connaissance, il faut le considérer, au contraire, comme un moyen de connaissance", La Production des connaissances scientifiques de l'administration, Presses de l'Université Laval (Québec), 1986).
}

11. Si les cas diffèrent, les soucis d'éthique demeurent, avec au premier chef celui de ne porter prejudice à aucun interlocuteur, ce qui n'est pas toujours aisé. Voir à ce sujet les travaux de la Société d'Anthropologie Appliquée du Canada ("Débat sur un code de déontologie professionnelle", Anthropologies et Sociétés, vol. 8, n $3: 117-129$ ), Université Laval (Québec), 1984. 
l'organisation des communications entre différents secteurs et entre niveaux hiérarchiques (12).

En définitive, la controverse concernant le caractère appliqué ou non de l'anthropologie industrielle vient du statut de ses objets dans la société moderne : lieux de production des opérateurs sociaux fondamentaux de notre société - les biens de consommation - et lieux de pouvoir, les entreprises et les administrations ne se laissent pas pénétrer facilement ; il faut montrer patte blanche et mener de longues négociations pour gagner l'accès aux ateliers et aux bureaux. Rien d'étonnant alors à ce que l'institution demande à y trouver son compte d'une manière ou d'une autre; l'important étant que le chercheur, chemin faisant, puisse y rassembler des matériaux pertinents pour l'étude de ces groupes humains qui ont tout pour séduire l'ethnologue : hiérarchisation, segmentation, division des tâches, des temps et des costumes... Certains premiers résultats semblent montrer que l'ethnologie ne peut qu'y gagner, et que, loin de trahir ses pères fondateurs, elle est particulièrement bien outillée pour interpréter l'histoire et l'actualité des objets centraux de notre modernité (13).

12. Il est étonnant de voir la facilité avec laquelle certains considèrent l'occupation d'un poste de travail comme le passage obligé de l'anthropologie dans l'entreprise. Dans l'ethnologie "exotique", le chercheur accompagnait certes les indigènes mais ne chassait et ne péchait pas forcément à leur place...

13. Cf. Georges BALANDIER, Le détour, Paris, Fayard, 1985 ; Françoise ZONABEND, 1989, La Presqu'tle au nucléaire, 1989, Paris, Odile Jaboc; Marc ABELES, Jours tranquilles en 1989, Paris, 1989, Odile Jacob ; et le Workshop "New Direction in Anthropological Research: Moving from Periphery to Center", qui s'est déroulé à la New-York University en mars 1990. 
Ce numéro a été coordonné par Monique Sélim et Kurumi Sugita.

Nous tenons à remercier les auteurs des manuscrits qui nous sont envoyés et qui sont autant de contributions à la vie de l'Association.

La forme et le contenu des articles n'engagent que la responsabilité de leurs auteurs. 


\title{
COLLECTIF DE REDACTION
}

\author{
Marie-Christine ANEST-COUFFIN \\ Claude ARDITI \\ Rita CORDONNIER \\ Nicole ECHARD \\ Marie-Paule FERRY \\ Jeanine FRIBOURG \\ Marc-Eric GRUENAIS \\ Danièle KINTZ \\ Annie LE PALEC \\ Olivier MASCLET \\ Catherine QUIMINAL \\ Monique SELIM \\ Alex-Louise TESSONNEAU
}

Toute correspondance doit être adressée à :

Journal des anthropologues

A.F.A. - E.H.E.S.S.

1 , rue du 11 Novembre

92120 MONTROUGE

Tél. : (1) 40921730

Répondeur

Permanence le mardi après-midi 


\section{SOMMAIRE}

\section{DOSSIER - ETHNOLOGIE DE L'ENTREPRISE}

Introduction M. SELIM et K. SUGITA

Entretien avec Gérard ALTHABE M. SELIM

Insertions ethnologiques dans l'entreprise M. SELIM De l'anthropologie des travailleurs à l'anthropologie de l'entreprise ....J. COPANS Anthropologie et industrie D. GUIGO Une expérience d'ethnographie en entreprise M. JEUDY-BALLINI Entretien avec Emmanuel DESVEAUX M. SELIM Ethnologie en entreprise B. MAURINES

Immersion, identification, quelle méthodologie appliquer

à l'observation des entreprises ?

K. POIRIER

Posture d'enquête: Une double contrainte N. HUGOT

Histoire et représentations d'une recherche en

secteur psychiatrique P.-N. DENIEUIL

Culture et projet d'entreprise P. TROMPETTE

L'entreprise brésilienne, lieu privé, lieu public R. CABANES

Taylorisme : une organisation aux multiples visages culturels K. SUGITA

Socio-anthropologie du travail P. BOUVIER

\section{L'ETHNOLOGIE AU JOUR LE JOUR}

Perspectives après la guerre du Golfe M. ARKOUN

La crise du Golfe : tensions et médiations culturelles J. HANNOYER et H. MONNA Les anthropologues dans la tourmente : la controverse sur les

quotas d'embauche en Inde

G. HEUZE

\section{ANTHROPOLOGIE VISUELLE}

Société Française d'Anthropologie Visuelle

En bref, dernière minute B. GANNE et J.-P. PENARD

Critiques de films ethnologiques

\section{NOUYELLES DE LA PROFESSION}

Des mots sur/de l'écologie

D. DORY

La recherche en sciences sociales et l'image :

le pays lobi

M. FIELOUX et J. LOMBARD A propos de l'article : "Pourquoi ferions-nous système ?" ....P. LABURTHE-TOLRA En écho à Philippe LABURTHE-TOLRA M.-P. FERRY L'anthropologie médicale en Europe. Etat de la question et perspectives M.-E. GRUENAIS Où en est l'anthropologie médicale appliquée? M.-E. GRUENAIS 
